\title{
Inverse modeling of flow tomography experiments in fractured media
}

\author{
Maria V. Klepikova, ${ }^{1}$ Tanguy Le Borgne, ${ }^{1}$ Olivier Bour, ${ }^{1}$ and Jean-Raynald de Dreuzy ${ }^{1,2}$ \\ Received 5 March 2013; revised 15 July 2013; accepted 30 September 2013; published 6 November 2013.
}

[1] The accurate characterization of the location, hydraulic properties, and connectivity of major fracture zones is essential to model flow and solute transport in fractured media. Cross-borehole flowmeter tests, which consist of measuring changes in vertical borehole flows when pumping a neighboring borehole, were shown to be an efficient technique to provide information on the properties of the flow zones that connect borehole pairs. The interpretation of such experiments may, however, be quite uncertain when multiple connections exist. In this study, we explore the potential of flow tomography (i.e., sequential cross-borehole flowmeter tests) for characterizing aquifer heterogeneity. We propose a framework for inverting flow and drawdown data to infer fracture connectivity and transmissivities. We demonstrate that successively exchanging the roles of pumping and observation boreholes improves the quality of available information and reduces the under-determination of the problem. The inverse method is validated for several synthetic flow scenarios. It is shown to provide a good estimation of connectivity patterns and transmissivities of main flow paths. It also allows the estimation of the transmissivity of fractures that connect the flow paths but do not cross the boreholes, although the associated uncertainty may be high for some geometries.

Citation: Klepikova, M. V., T. Le Borgne., O. Bour, and J.-R. de Dreuzy (2013), Inverse modeling of flow tomography experiments in fractured media, Water Resour. Res., 49, 7255-7265, doi:10.1002/2013WR013722.

\section{Introduction}

[2] Fractured aquifers are characterized by strong heterogeneities at multiple scales [e.g., Bonnet et al., 2001]. However, flow generally takes place in a limited number of dominant fracture zones [Tsang and Neretnieks, 1998]. The identification of these dominant flow paths, their connectivity patterns and their hydraulic properties is critical as they control the transfer of fluids as well as the transport of solutes in the subsurface [Dorn et al., 2011; Illman et al., 2009].

[3] Recent developments show that applying hydraulic tomography (HT) to cross-hole pumping test is a promising approach to delineate hydraulic heterogeneities [Yeh et al., 2000; Sharmeen et al., 2012]. The principle of such tomography is to change successively the pumping and monitoring wells to image the properties of the medium [Butler et al., 1999]. Thus, Hao et al. [2008] demonstrated the feasibility of hydraulic tomography to detect high hydraulic conductivity fracture zones and obtain their general connectivity pattern. Using this method, Illman et al. [2009] were able to successfully identify and characterize

\footnotetext{
${ }^{1}$ Géosciences Rennes, OSUR, UMR CNRS 6118, University of Rennes 1, Rennes, France.

${ }^{2}$ Institute of Environmental Analysis and Water Studies, CSIC, Barcelona, Spain.

Corresponding author: T. Le Borgne, Géosciences Rennes, OSUR, UMR CNRS 6118, University of Rennes 1, Rennes CEDEX, France. (tanguy.le-borgne@univ-rennes1.fr)

C2013. American Geophysical Union. All Rights Reserved. 0043-1397/13/10.1002/2013WR013722
}

transmissive flow paths in a fractured granite where all transmissive fractures were isolated with a packer system. However, isolation of fractures with a packer system involves a large amount of equipments and is practically impossible in many cases, in particular when boreholes are screened. Moreover, to ensure the effective location of measurement intervals, the identification of flowing fractures is required a priori.

[4] An alternative method that does not require the use of packers is the cross-borehole flowmeter test approach [Paillet, 1998; Le Borgne et al., 2006b]. The principle of this approach is to monitor vertical borehole flows induced by differences in fracture hydraulic heads. These differences in heads are either driven by ambient conditions or by pumping in neighboring boreholes. Pumping in one of the wells induces hydraulic head variations in large-scale flow paths, which in turn drives vertical flow variations between the fractures intersecting the observation borehole [Le Borgne et al., 2006a]. These flow variations can be interpreted to estimate the connectivity and hydraulic properties of the fracture zones that connect borehole pairs [e.g., Paillet, 1998, 2000; Le Borgne et al., 2007; Paillet et al., 2012]. Nevertheless, due to the many degrees of freedom, matching the cross-borehole flowmeter data with a forward model that incorporates flow path geometry and hydraulic properties as well as borehole is unlikely to have neither a simple nor a unique solution. As yet, no inverse methods have been developed for interpreting cross-borehole flowmeter tests. This is the objective of the present study.

[5] We investigate the interest of using the cross-borehole flowmeter test principle in a tomographic approach, where the pumping and observation wells are successively 
changed. We hypothesize that such tomography approach based on flowmeter measurements should reduce the uncertainty and nonuniqueness of the solution with respect to classical cross-borehole flowmeter tests. Furthermore, it should provide the same level of characterization as 3-D hydraulic tomography without the necessity of using packers. We therefore develop an inverse modeling framework for flow tomography experiments using a discrete fracture conceptual model of flow and connectivity.

[6] In section 2 of this paper, we detail the principle of the cross-borehole flowmeter test. In section 3, we present a simple conceptual model of flow and connectivity for fractured media. In section 4, we describe the numerical flow model, and in section 5 we develop an inverse problem approach for flow tomography. Finally, we focus on a sensitivity analysis, discuss the uncertainties of the method for different flow scenarios and multiple connection fracture networks.

\section{Principle of Cross-Borehole Flowmeter Tests}

[7] An example of flow pattern for two boreholes under ambient, single-borehole and cross-borehole pumping conditions is shown in Figure 1. Under ambient condition, differences in hydraulic heads between the different flow paths that connect to a borehole generally create ambient vertical flow within the borehole [Paillet, 1998]. During pumping in one of the wells, hydraulic head changes occur only in the flow paths connected to the pumping well. By inspection of the pairs of ambient and pumping flow profiles, the main flow zones intersecting each of the boreholes can be detected and characterized from changes in the measured vertical flow [Paillet, 1998; Williams and Paillet, 2002; Le Borgne et al., 2007]. This single-borehole flowmeter method allows thus identifying the main flowing fractures in each borehole as well as their transmissivities in the near field around borehole. During cross-borehole flowmeter tests the drawdown and the variations in vertical velocity $(s, v)$ are monitored in observation boreholes. The drawdown $s$ depends on the overall transmissivity of all connected fractures. The magnitude of the velocity $v$ is controlled by the difference in hydraulic heads induced by pumping. This difference in

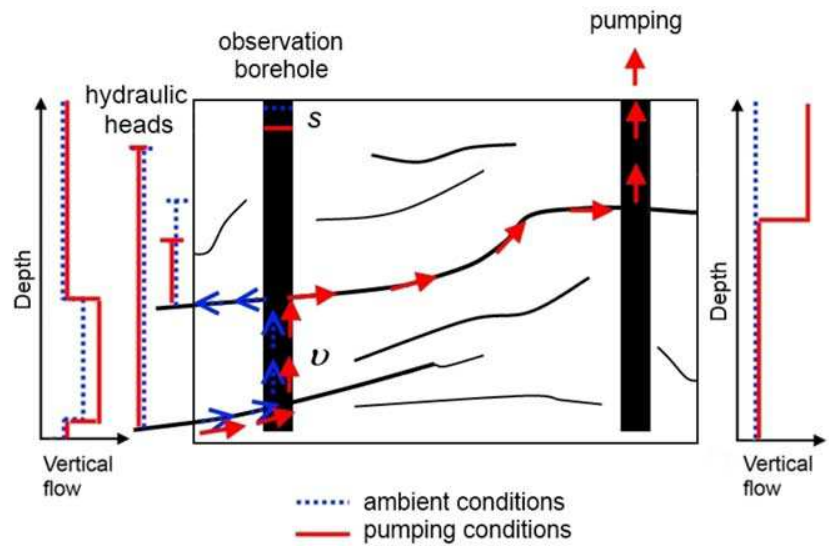

Figure 1. Flow pattern, hydraulic head difference between fractures and flow profiles for a pair of pumping and observation boreholes connected by one main flow path under ambient (blue dotted line) and pumping (red line) flow conditions. hydraulic heads, in turn, depends on the transmissivities of the connecting fractures. Cross-borehole flowmeter tests can be used to determine which of the fracture zones in the observation well are connected to the pumping well and to infer fracture hydraulic properties through type curves matching [Hess, 1986; Molz et al., 1989; Paillet, 1998]. Nevertheless, if multiple connections exist between boreholes, the connectivity patterns may be less easy to identify as different combinations of fracture connectivity and transmissivity may provide similar cross-borehole flow responses [Le Borgne et al., 2007]. In the following, we investigate this uncertainty and its potential reduction by use of the tomography approach.

\section{Conceptual Model of Flow and Connectivity}

[8] Appropriate inverse models to interpret hydraulic tomography data sets are still under debate [e.g., Day-Lewis et al., 2000; Yeh et al., 2000; Illman et al., 2008, 2009; Brauchler et al., 2011; Castanga et al., 2011]. Classical approaches assign averaged properties to bulk regions of the aquifer and results in smooth tomograms that do not match the sharp variations of fracture patterns [Day-Lewis et al., 2005; Illman et al., 2009]. In the case of fractured media, discrete fracture network models may be more appropriate since flow is highly localized in fractures with hydraulic apertures of a few millimeters to at most a centimeter [Olsson, 1992; Wellman and Poeter, 2005]. However, to express the complexity of real fracture geometry (Figure 2a), a large number of adjustable parameters including fracture location, orientation, length, and spacing of fractures would be required. Considering a typically moderate number of wells and because of the decreasing sensitivity of hydraulic data with the distance from the borehole, the data available in the field are generally insufficient to provide such a degree of complexity [Le Goc et al., 2010]. For the inverse problem to be well posed, either using additional constraints such as geophysical surveys [e.g., Day-Lewis et al., 2003; Dorn et al., 2012] or drastic simplifications are required.

[9] Considering a pumping and an observation borehole, several main flow paths can in general be detected in each borehole with single-borehole flowmeter measurements (Figure 2a). Considering the case where each borehole is intersected by two main fractures, the typical fracture connections that are possible can be synthesized through (Figure 2b): (1) a single fracture connecting the pumping and observation boreholes and one disconnected fracture in each borehole; (2) two pumping borehole fractures connecting one observation borehole fracture; (3) two fractures connecting both boreholes without any interconnection between them; and (4) two fractures connecting both boreholes with an interconnection between them through a fracture that does not intersect the boreholes. Furthermore, we believe that more complex cases with a large number of fractures in the interval between the boreholes can be approximated by juxtaposition of these basic kinds of connections.

[10] Here we propose a simplified discrete fracture network approach that highlights such connectivity structures. This conceptual model attempts to reproduce fracture network connectivity without taking fracture geometry (length, orientation, dip) into account. In this simplified fracture network model (Figure 2c), the observation and 
a

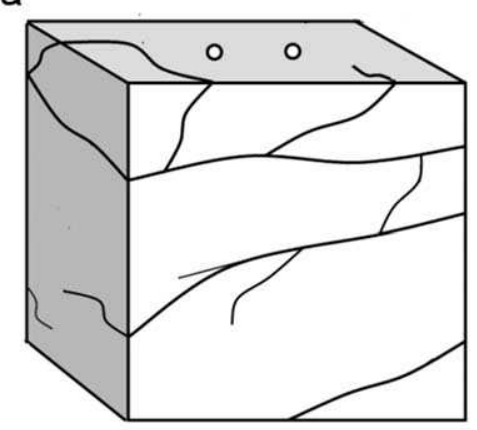

b 1.

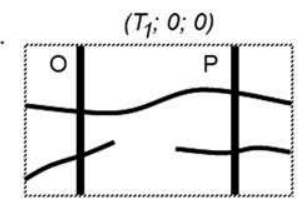

$\left(T_{1} ; T_{2} ; 0\right)$

2.

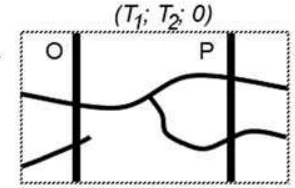

3.

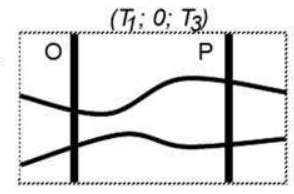

$\left(T_{1} ; T_{2} ; T_{3}\right)$

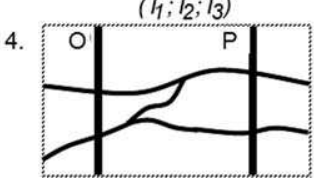

C $\left(T_{1} ; T_{2} ; T_{3}\right)=($ param $1 ;$ param 2 ; param 3$)$

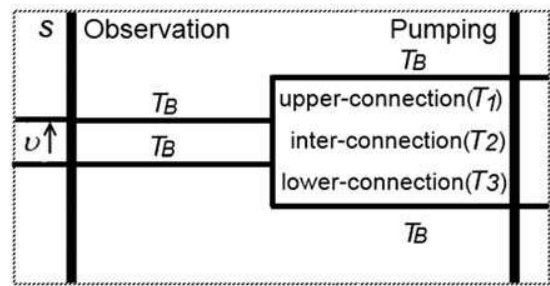

Figure 2. (a) Example of fracture network intersected by two boreholes. (b) Cross sections of synthetic model: the observation and pumping boreholes are both intersected by two flowing horizontal fractures of equal transmissivity $\left(T_{B}\right)$. These fractures are connected by a vertical fracture consisting of three sections with $T_{1}, T_{2}$, and $T_{3}$ transmissivities. (c) Basic connections that could be modeled by different combinations of connection transmissivities $T_{1}, T_{2}$, and $T_{3}$.

pumping boreholes are both intersected by two horizontal fractures of transmissivity $T_{B}$. These fractures are connected by a vertical fracture equally distanced from both boreholes. To control connectivity between the boreholes different values of transmissivity are attributed to the different sections of the vertical connecting fractures $\left(T_{1}, T_{2}\right.$, and $T_{3}$ in Figure $\left.2 \mathrm{c}\right)$. The upper $\left(T_{1}\right)$ and/or lower $\left(T_{3}\right)$ sections of the vertical fracture ensure the connectivity between both boreholes, while the interconnection $\left(T_{2}\right)$ section controls the interconnection of the upper and lower fractures. The different combinations of these parameters $\left(T_{1}, T_{2}\right.$, and $\left.T_{3}\right)$ allow this case to be split into the four kinds of fracture connections described in Figure 2b. Thus, this approach allows all the principal types of connections to be differentiated (Figure 2b) while introducing an order of complexity that matches the information content of the data. In applying this simplified model to real fracture systems, it is important to recognize that despite that the approach does not model fracture network geometry, we aim to asses both the connectivity of preferential flow paths and their hydraulic properties. We believe that our conceptual model is better adapted for fractured media compared to classic continuous descriptions, as it represents the discrete nature of fracture networks and the long range spatial correlation of these structures. Furthermore, this simplified representation is consistent with the information content of cross-borehole hydraulic tests.

[11] The flow tomography approach proposed in this study consists of the following steps:

[12] 1. Detection of fracture zones intersecting the observation and pumping boreholes and definition of local transmissivities of these fractures $\left(T_{B}\right)$ through interpretation of single-borehole ambient and steady pumping flow profiles [Paillet, 2000; Sawdey et al., 2012].
[13] 2. Definition of the fracture connectivity model. Once the number of flowing fractures has been determined in each borehole from the previous step, the connectivity between these flow zones is simply a combination of the total number of fractures. The number of parameters is equal to $n-1$ where $n$ is the number of flowing fractures. Their spatial arrangement still depends on the respective number of flowing fractures from each borehole.

[14] 3. Estimation of the transmissivities of connecting fractures between the pumping and observation boreholes through inversion of cross-borehole flow profiles.

[15] The first two steps correspond to preliminary investigations and in this study we are focusing on the inverse problem of the last step.

\section{Direct Flow Modeling}

[16] We study the hydraulic responses of connected fractures under cross-borehole pumping conditions, by developing a 3-D numerical model (with 2-D flow in each fracture) that simulates flow in the simplified fracture network (Figure 3 ). The model considers cylindrical boreholes that are intersected by the fractures. The rock matrix between fractures is impermeable but the approach can be easily extended to fractured/porous rock. We assume a Darcy flow in the fractures, and the volume flow rate per unit fracture length on the fracture is given by

$$
u=-\frac{k}{\mu} d \nabla p
$$

where $k$ describes the fracture permeability $\left(\mathrm{m}^{2}\right), d$ is the fracture aperture $(\mathrm{m})$. Each fracture is characterized by a value of transmissivity $T$, which is given by 


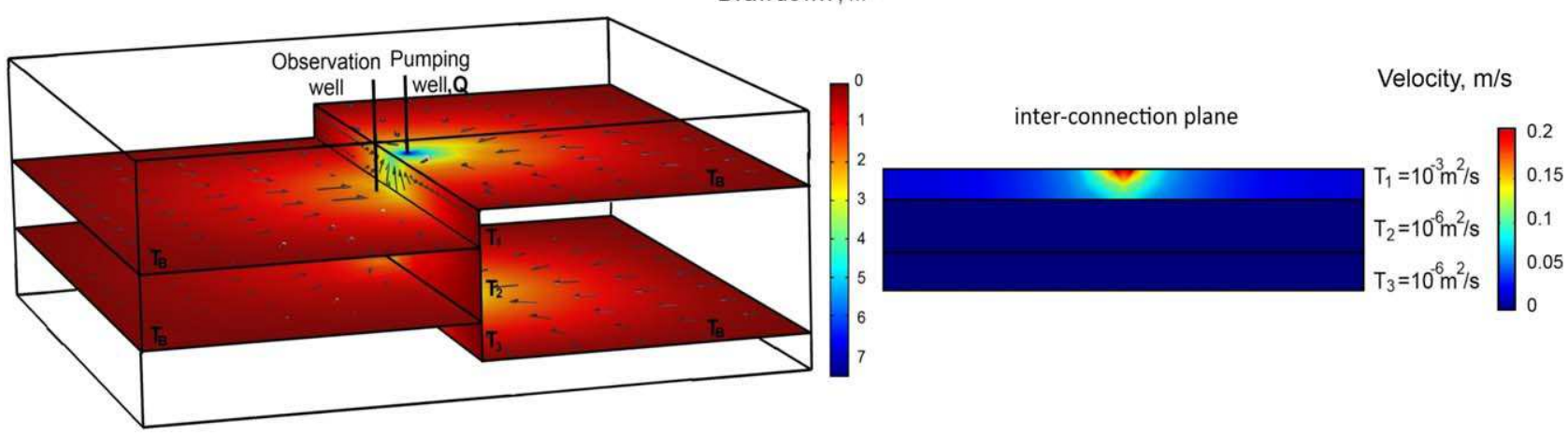

Figure 3. (a) Drawdown $s$ (color scale) and flow velocity field $v$ (arrows) in an example of flow model geometry. For this example the borehole radius is $0.05 \mathrm{~m}$, the pumping rate is $Q=2.5 \cdot 10^{-3} \mathrm{~m}^{3} / \mathrm{s}$. The transmissivities of fractures are given by $T_{B}=5 \cdot 10^{-4} \mathrm{~m}^{2} / \mathrm{s}, T_{1}=10^{-3} \mathrm{~m}^{2} / \mathrm{s}, T_{2}=10^{-6} \mathrm{~m}^{2} / \mathrm{s}$, and $T_{3}=10^{-6} \mathrm{~m}^{2} / \mathrm{s}$. (b) Distribution of the flow velocity $v$ in the interconnection plane.

$$
T=d \frac{k \rho g}{\mu} .
$$

[17] In the following synthetic flow models, the fracture aperture is fixed at $d=1 \cdot 10^{-3} \mathrm{~m}$. We apply zero-head boundary conditions on the edges of fractures and no-flux boundary on the faces of the rock matrix. Under these conditions, no ambient flow takes place in the boreholes. Hence, the model results can be compared to field data, with the ambient flow profile substracted from the pumping profiles [Paillet, 1998]. Furthermore, we simulate here steady state flow profiles, which are relevant for fracture media where quasi-steady state conditions are quickly attained, due to low storativity and relatively large transmissivity [Le Borgne et al., 2007]. However, the approach can be further extended to simulate and invert transient responses. The partial differential equations are solved with the finite element code Comsol Multiphysics 4.2a with a fine tetrahedral meshing. Various tests were performed to get a mesh-independent result. The code was also benchmarked against analytical solutions for ground water flow and an existing hydraulic model for cross-borehole flow analysis [Paillet, 1998].

[18] The rectangular domain $L_{x}=200 \mathrm{~m}, L_{y}=200 \mathrm{~m}$, $L_{z}=100 \mathrm{~m}$ dimensions are set to be much larger than the radius of influence of the pumping tests. Two boreholes separated by $7 \mathrm{~m}$ are each intersected by a pair of fractures. Pumping in one of the wells induces flow through connected fractures. The borehole radius is set at $0.05 \mathrm{~m}$ and the pumping rate is fixed at $Q=2.5 \cdot 10^{-3} \mathrm{~m}^{3} / \mathrm{s}$. To test the model, we let the transmissivities of the fracture connections vary in a reasonable range given by $T_{n} \simeq$ $\left[10^{-6} \mathrm{~m}^{2} / \mathrm{s}, 10^{-3} \mathrm{~m}^{2} / \mathrm{s}\right]$ for $n=1,2$, and 3. For illustration purposes, the transmissivities of the fractures intersecting the borehole are set equal and fixed at an intermediate value $T_{B}=5 \cdot 10^{-4} \mathrm{~m}^{2} / \mathrm{s}$. Nevertheless, they can in general be different and should be determined from the singleborehole profiles [Paillet, 1998; Le Borgne et al., 2006a]. An example of flow simulation is given in Figure 3 , for the case where the upper connection section is the most permeable $\left(T_{1}=10^{-3} \mathrm{~m}^{2} / \mathrm{s}, T_{2}=10^{-6} \mathrm{~m}^{2} / \mathrm{s}, \quad\right.$ and $T_{3}=$ $10^{-6} \mathrm{~m}^{2} / \mathrm{s}$ ). The inverse model consists of adjusting the transmissivities of the connecting fracture to match the ver- tical flow velocities and borehole drawdowns. The transmissivities of the connecting fractures $\left(T_{1}, T_{2}\right.$, and $\left.T_{3}\right)$ are the only fitted parameters. The inverse method for this task is discussed in the following section.

\section{Inverse Model Description}

[19] To estimate the fracture transmissivities from the cross-borehole flow profiles and drawdown measurements in an inverse problem approach we couple the direct flow model with an optimization algorithm. We adopt a quasiNewton algorithm which is effective for solving nonlinear optimization problems [e.g., Yeh, 1986; Cheng and Yeh, 1992; Tarantola, 2004]. A sequential quadratic programming (SQP) constrained algorithm [Mayer et al., 1999] with quasi-Newton approximations to the Hessian matrix (i.e., of the second-order partial derivatives of the misfit function) provides a superior rate of convergence when compared to the classical gradient methods [Tarantola, 2004; Hill and Tiedeman, 2007]. The misfit function, FO, which evaluates the difference between flow model simulations and observations and includes the data misfit of drawdown data $\left(F O_{\text {drawdown }}\right)$ and velocity data $\left(F O_{\text {velocity }}\right)$, is given by

$$
\begin{gathered}
F O=F O_{\text {drawdown }}+F O_{\text {velocity }}=\frac{1}{\sigma_{s}^{2}} \frac{1}{N_{s}} \sum_{0}^{N_{s}}\left(s_{o b s}-s_{\text {mod }}\right)^{2} \\
+\frac{1}{\sigma_{v}^{2}} \frac{1}{N_{v}} \sum_{0}^{N_{v}}\left(v_{\text {obs }}-v_{\text {mod }}\right)^{2}
\end{gathered}
$$

where $v_{o b s}$ and $s_{o b s}$ are the flowmeter and drawdown observations, $\sigma_{v}$ and $\sigma_{s}$ are standard deviations of errors for flow and drawdown, respectively, $N_{v}$ and $N_{s}$ are the numbers of observations for flow and drawdown, respectively, $v_{\text {mod }}$ and $S_{\text {mod }}$ are the velocity and drawdown predicted by the model. As demonstrated in section 6.3.1, for the case with two fractures (Figure 2b) the objective function thus defined has several local minima (Figure 7). The number of local minima tends to increase with the number of fractures. Because the result provided by the quasi-Newton method is not necessarily the absolute minimum of the objective function, we 
improve this direct-search method by using the global search algorithm from the MATLAB optimization toolbox to generate a number of random starting points [Urgay et al., 2007]. A local solver is then used to find the optima in the basins of attraction of the starting points. In the next section, we show that this simple improvement is well adapted to the fracture models studied here. As the objective function (equation (3)) is normalized to the data errors, data errors influence the value of the objective function. We then assume that all the solutions with the objective function value less than one are acceptable.

\section{Results}

[20] A series of numerical simulations is first performed to study the sensitivity of observation borehole drawdown and vertical flow velocity $(s, v)$ with respect to parameters. These results are then used to discuss the uncertainties in parameter estimation related to measurement errors. Finally, to validate our inverse approach, the flow tomography inversion is performed for different synthetic fracture network geometries.

\subsection{Sensitivity Analysis}

[21] We investigate the sensitivity of drawdown and vertical flow velocity in observation borehole $(s, v)$ with respect to the log-transformed transmissivities of connecting fractures. To do so, we simulate flow in the simplified fracture network (Figure 2c) with fixed transmissivity of the fractures intersecting the borehole $\left(T_{B}=5 \cdot 10^{-4} \mathrm{~m}^{2} / \mathrm{s}\right)$ and varying the connecting fracture transmissivities $\left(T_{1}, T_{2}\right.$, and $T_{3}$ ). Drawdown and vertical borehole velocities were obtained for all the combinations of transmissivities of connecting fractures changing in the interval $T_{n} \simeq$ $\left[10^{-6} \mathrm{~m}^{2} / \mathrm{s}, 10^{-3} \mathrm{~m}^{2} / \mathrm{s}\right]$ for $n=1,2$, and 3 with a step $\log T=0.2$ (Figure 2c).

\subsubsection{Sensitivity of Drawdown to Connecting Fracture} Transmissivity

[22] Drawdown values obtained for the different fracture transmissivities are presented in Figure $4 \mathrm{a}$ as orthogonal slice planes along the $\log T_{1}, \log T_{2}$, and $\log T_{3}$ directions. The drawdown in the observation well is found to be strongly sensitive to the upper and lower connection trans- missivities $\left(T_{1}\right.$ and $\left.T_{3}\right)$ and not very sensitive to the interconnection fracture transmissivity $T_{2}$. Figure $4 \mathrm{~b}$ presents a slice with a fixed value of interconnection transmissivity $\log T_{2}=-3$. An increase in upper $\left(T_{1}\right)$ or lower $\left(T_{3}\right)$ connection transmissivity results in an increase in drawdown. Due to the symmetry of the system, the upper $\left(T_{1}\right)$ and lower $\left(T_{3}\right)$ connection transmissivities have equal effects on the drawdown in the observation well, as shown by the symmetry of the drawdown map in Figure 4b.

\subsubsection{Sensitivity of Flow Velocity to Connecting} Fracture Transmissivity

[23] The influence of fracture transmissivities on vertical borehole velocity is shown in the $3-\mathrm{D}$ matrices in Figure 5a. A 2-D slice with a fixed interfracture transmissivity $\log T_{2}=-6$ is shown in Figure 5b. The upflow is maximum when the upper connection transmissivity $T_{1}$ is largest and the lower connection transmissivity $T_{3}$ is smallest. The distribution of hydraulic head for this configuration is shown in Figure 3. Conversely, the downflow is maximum when the upper connection transmissivity $T_{1}$ is smallest and the lower connection transmissivity $T_{3}$ is largest. When the difference in hydraulic heads between the upper and lower connections decreases, the value of absolute velocity decreases. Hence, the difference between the upper $\left(T_{1}\right)$ and lower $\left(T_{3}\right)$ connections transmissivities controls the absolute velocity value. The direction of the vertical velocity is toward the largest transmissivity connection.

[24] The effect of the interconnection transmissivity $T_{2}$ can be understood from the slice with the fixed transmissivity of the lower connection $\log T_{3}=-6$ (Figure 5c). An increase of fracture interconnection transmissivity $T_{2}$ leads to a decrease in the absolute value of vertical velocity since it corresponds to a decrease in the difference in hydraulic heads between the upper and lower connections.

6.1.3. Synthesis of the Sensitivity Analysis

[25] The results of the sensitivity analysis can be summarized as follows:

[26] A. The observed well drawdown increases with the upper $\left(T_{1}\right)$ or lower $\left(T_{3}\right)$ connection transmissivity and is insensitive to the interconnection transmissivity $T_{2}$.

[27] B. The magnitude of the vertical borehole flow velocity increases with the difference between the upper $\left(T_{1}\right)$
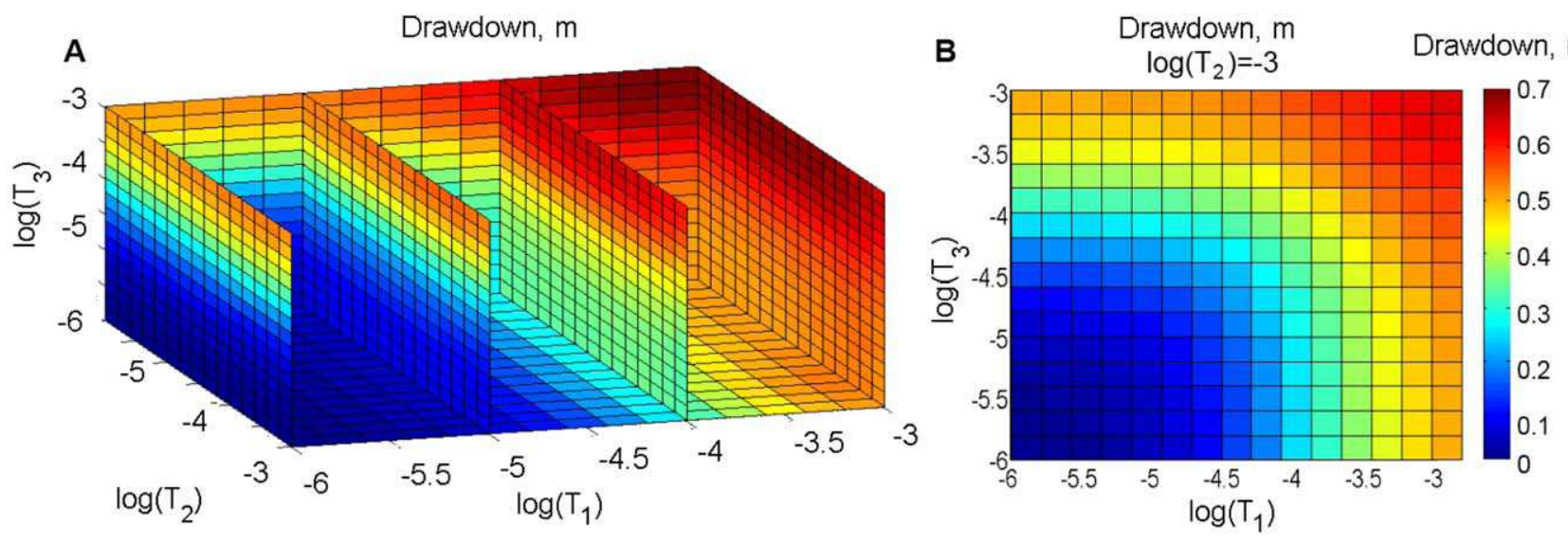

Figure 4. (a) Model computation of drawdown $s$ in the observation borehole as a function of the logtransformed transmissivities of the vertical fractures $\log T_{1}, \log T_{2}$, and $\log T_{3}$. (b) Model computation of drawdown $s$ in the observation borehole with fixed interconnection fracture transmissivity $\log T_{2}=-3$. 


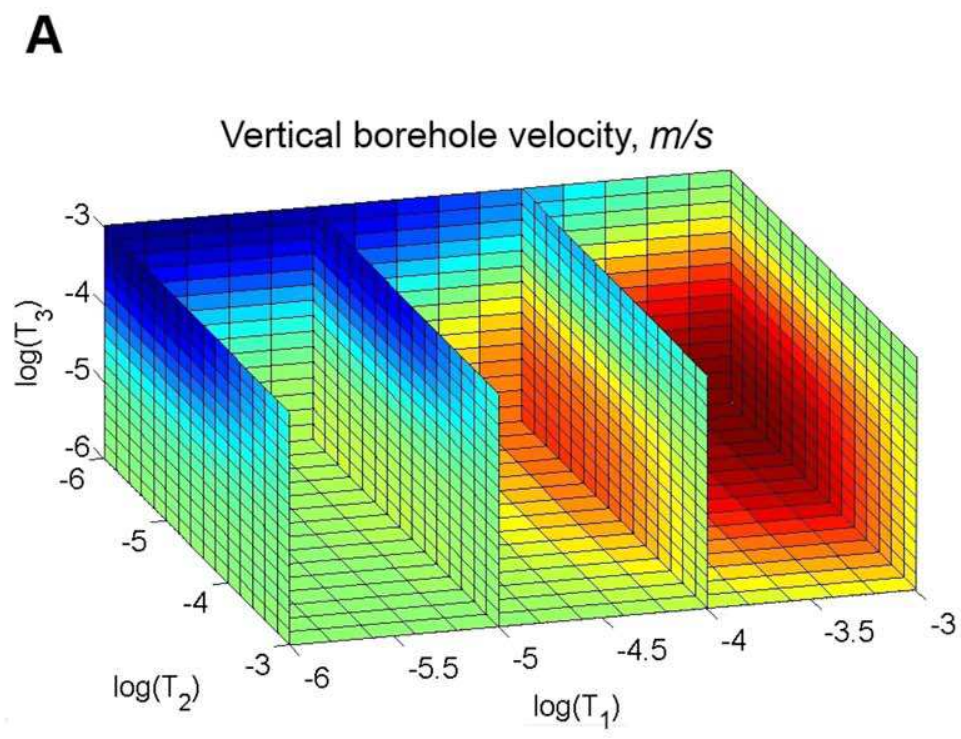

B Vertical borehole velocity,
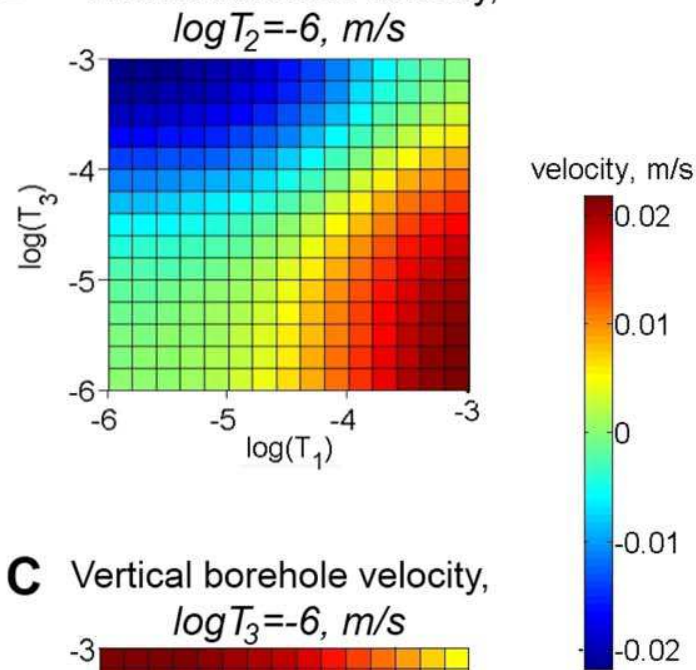

Figure 5. (a) Model computation of vertical flow velocities $v$ in observation borehole as a function of $\log$-transformed transmissivities of vertical fractures $\log T_{1}, \log T_{2}$, and $\log T_{3}$. (b) Model computation of vertical flow velocities $v$ in the observation borehole with fixed interconnection fracture transmissivity $\log T_{2}=-6$. (c) Model computation of vertical flow velocities $v$ in the observation borehole with fixed transmissivity of the lower connection $\log T_{3}=-6$.

and lower $\left(T_{3}\right)$ connection transmissivities and decreases with the interconnection transmissivity $T_{2}$.

[28] C. The direction of the vertical borehole flow velocity is toward the largest connection transmissivity.

[29] These conclusions constitute the major constraints provided by flow and borehole drawdown measurements.

\subsection{Uncertainty Analysis}

[30] To evaluate the capacity of flow tomography experiments to provide reliable estimates of fracture transmissivity, we perform an uncertainty analysis on the basis of the previously obtained results (Figures $4 \mathrm{a}$ and $5 \mathrm{a}$ ). The uncertainty is determined for each pair of observations as the standard deviation of the parameters that provide similar solutions within the range of errors $\left(\sigma=\left(\sigma_{s}, \sigma_{v}\right)\right)$. Figure 6 presents the estimated uncertainties for a measurement errors $\sigma=\left(0.01 \mathrm{~m}, 2 \cdot 10^{-3} \mathrm{~m} / \mathrm{s}\right)$, which correspond to typical measurement errors [Klepikova et al., 2011]. The white areas correspond to precluded couples $(s, v)$ for the range of transmissivity values considered here.

[31] According to Figures $6 \mathrm{a}$ and $6 \mathrm{c}$ the uncertainty associated with the upper $\left(T_{1}\right)$ and lower $\left(T_{3}\right)$ connection transmissivities is smaller when flow is directed toward these connections in the observation well. This result can be understood from the sensitivity analysis (section 6.1.3). The fracture zone toward which the flow is directed in the observation well corresponds to the largest transmissivity connection (conclusion C). This connection is thus the one that controls drawdown in the observation well (conclusion A). Hence, its value is well determined. Conversely, the uncertainty for the connection that produces the flow in the observation well increases as the magnitude of borehole flow decreases. For small borehole flows, similar velocities can be produced by different combinations of the interconnection transmissivity $T_{2}$ and the smallest values of $T_{1}$ and $T_{3}$ (conclusion B).

[32] The uncertainty pattern for fracture interconnection transmissivity $T_{2}$ in Figure $6 \mathrm{~b}$ shows higher uncertainty for smaller borehole flow velocities in the observation well. This uncertainty decreases with the increase in amplitude of the vertical velocity. According to conclusion $\mathrm{B}$, the borehole flow velocities are small when the fracture connection transmissivities $T_{1}$ and $T_{3}$ are close to each other. Furthermore, in the latter case, they are not very sensitive to the fracture interconnection transmissivity $T_{2}$. Thus, for zero vertical velocity the uncertainty about the $T_{2}$ parameter is maximum. Hence, in the case of equal upper $\left(T_{1}\right)$ and lower $\left(T_{3}\right)$ connection transmissivity it is difficult to distinguish between the third and fourth kinds of fracture connection on Figure $2 b$. Note, that however the sensitivity could be improved by increasing the pumping rate. 

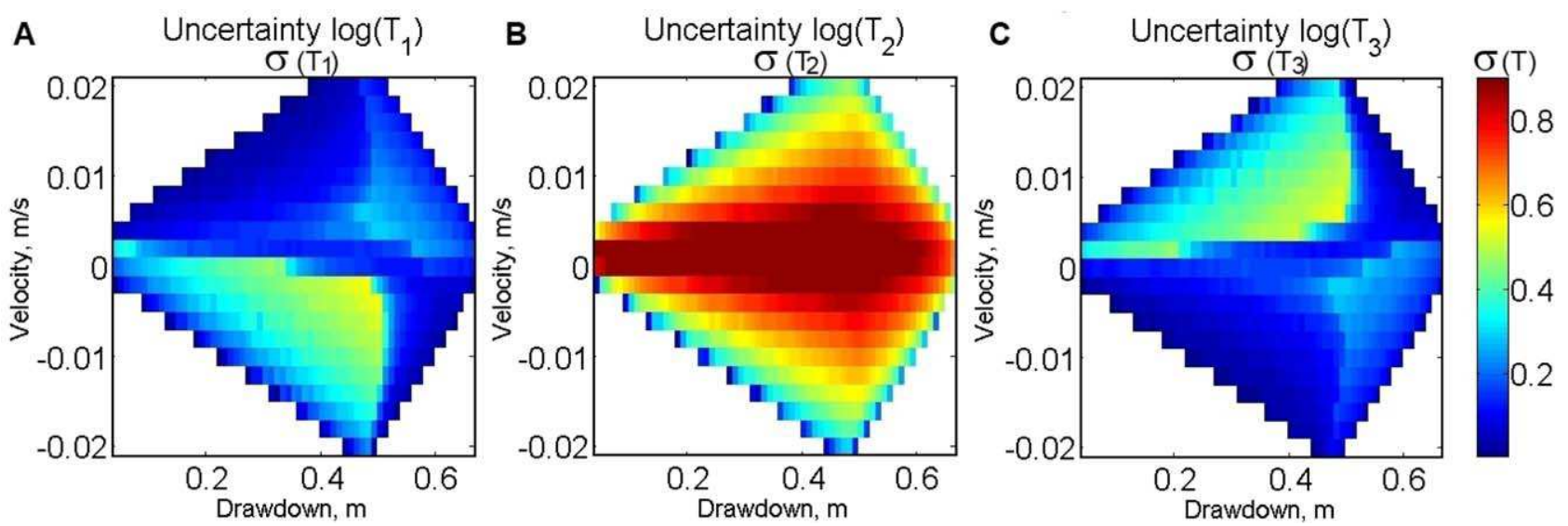

Figure 6. Uncertainty analysis of the flow model. The range of allowable drawdown and vertical borehole velocity observations $(s, v)$ for the synthetic case presented in Figure $2 \mathrm{~b}$ and the estimated uncertainties about (a) $\log T_{1}$, (b) $\log T_{2}$, and (c) $\log T_{3}$ for measurement errors $\left(\sigma_{s}, \sigma_{v}\right)=$ $\left(0.01 \mathrm{~m}, 2 \cdot 10^{-3} \mathrm{~m} / \mathrm{s}\right)$.

[33] The added value of the flow velocity data is determined by conducting the same study without taking into account vertical borehole velocity. Hence, in this case only the first constraint applies (conclusion A). The observation borehole drawdown is found to be almost insensitive to the interconnection transmissivity $T_{2}$. Moreover, the uncertainty about the $T_{1}$ and $T_{3}$ parameters is found to be higher and ranges from 0.3 to 0.9 , which is the maximum uncertainty for the range of transmissivities tested. Thus, uncertainty can be significantly reduced by using velocity data.

[34] Another possible source of errors is the uncertainty on local transmissivities of fractures intersecting the observation and pumping boreholes $\left(T_{B}\right)$. However, it is difficult to draw general conclusions on this uncertainty since it is likely to depend on relative transmissivities of these fractures. The most uncertain connection transmissivities, $T$ 's, are likely to be those that connect to low permeable $T_{B}$ 's, which transmit only weakly to the borehole any hydraulic head variations that may occur in the connected flow paths. Furthermore, we believe that in case of real fracture networks the main source of uncertainty probably comes from the simplification of the fracture network geometry.

\subsection{Inverse Modeling of Multiple Connection Fracture Networks}

\subsubsection{Simple Synthetic Cases}

[35] We first apply the inverse approach to the simple synthetic cases 1 and 4 of Figure 2b. For synthetic case 1, the parameter set is $\left(\log T_{1}, \log T_{2}, \log T_{3}\right)=(-6,-6,-3)$, which provides the largest possible vertical velocity $v=$ $-0.021 \mathrm{~m} / \mathrm{s}$ and a drawdown $s=0.5 \mathrm{~m}$ (Figures 4 and 5). For synthetic case 4 , the parameter set is $\left(\log T_{1}\right.$, $\left.\log T_{2}, \log T_{3}\right)=(-4.6,-4.6,-4.6)$, which provides a zero vertical velocity and a drawdown $s=0.27 \mathrm{~m}$. To underline the interest of combining drawdown and flow data we present the misfit of each data type separately, $F O_{\text {drawdown }}$ and $F O_{\text {velocity }}$, and the sum of the two misfits, which corresponds to the global objective (equation (3)).

[36] The objective functions for synthetic case 1 are shown in Figure 7 as a function of the log-transformed parameters. For a fixed upper connection transmissivity $\log T_{1}=-6$, the drawdown misfit $\left(F O_{\text {drawdown }}\right.$, Figure $\left.7 \mathrm{a}\right)$ displays few local minima. This is due to the insensitivity of the drawdown to the interconnection transmissivity $T_{2}$. For a fixed interconnection transmissivity $\log T_{2}=-6$, the drawdown misfit $F O_{\text {drawdown }}$ (Figure $7 d$ ) also presents several local minima since different combinations of $T_{1}$ and $T_{3}$ can give similar drawdowns. The velocity misfit on the other hand shows only one global minimum (Figures $7 \mathrm{~b}$ and $7 \mathrm{e}$ ). Hence, the global objective function presents one global minimum as well (Figures $7 \mathrm{c}$ and $7 \mathrm{f}$ ).

[37] The objective functions for synthetic case 4 are shown in Figure 8 as a function of the log-transformed parameters for a fixed interconnection $\log T_{2}=-4$.6. As in the previous case, the drawdown misfit $F O_{\text {drawdown }}$ possesses several local minima (Figure $8 \mathrm{a}$ ). In this case, the velocity misfit $F O_{\text {velocity }}$ also has a number of local minima lying on a diagonal (Figure 8b). This is explained by the fact that zero borehole flow velocity results from equivalent values of upper and lower connections (Figure 5). However, the sum of both misfits provides a convex function with a global minimum (Figure 8c). Note that we do not present here the misfit as a function of $\log T_{2}$ since both drawdown and velocity are insensitive to this parameter in this case (Figure 6b).

[38] In both cases the objective functions are convex and possess a global minimum. Hence, the global minima are found successfully by the applied optimization algorithm (section 5). Thus, the inverse method proposed seems to be sufficiently robust to identify the main fracture connectivities and transmissivities. In the following, we test the capacity of the flow tomography inversion to identify fracture flow patterns in more complex structures.

\subsubsection{Complex Synthetic Cases}

[39] In this section, we test the inverse approach for a synthetic example with more complex fracture network geometry. The cross section of model geometry is presented in Figure 9a (pumping and observation boreholes can be reversed). The pair of "pumping-observation" boreholes is intersected by six fractures of transmissivity $T_{B}$ forming two zones of constant vertical flow in each borehole. The system is parameterized by five fracture connection 

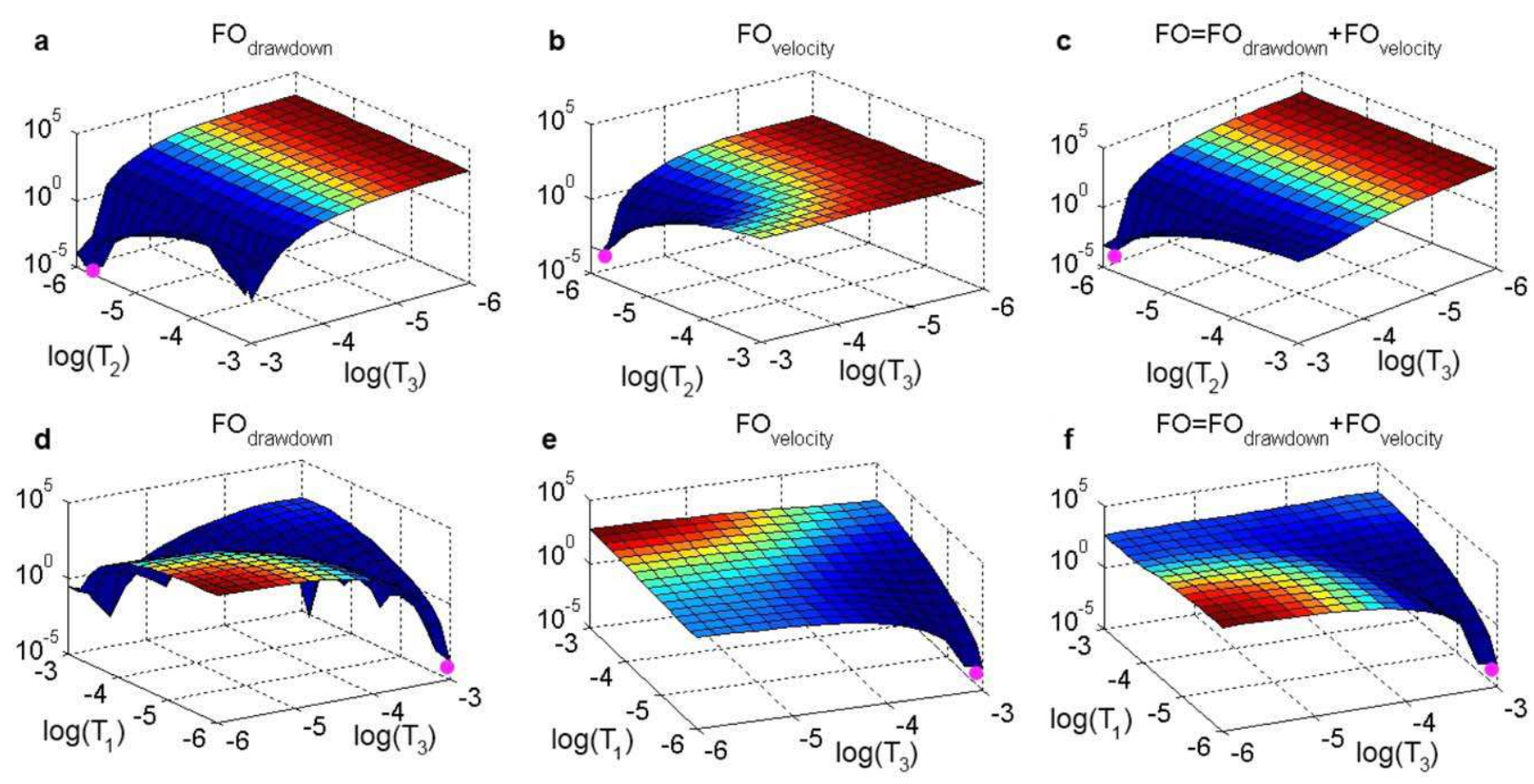

$\left[\log T_{1}, \log T_{2}, \log T_{3}\right]=[-6,-6,-3]$

Figure 7. The objective function for the simple synthetic case $\left(\log T_{1}, \log T_{2}, \log T_{3}\right)=(-6,-6,-3)$ (Figure 2) for the (a) drawdown, (b) velocity, and (c) the sum of both with respect to the log-transformed inter and lower connection transmissivities $\left(\log T_{2}\right.$ and $\left.\log T_{3}\right)$ and with respect to the $\log$-transformed upper and lower connection transmissivities $\left(\log T_{1}\right.$ and $\left.\log T_{3}\right)(\mathrm{d}-\mathrm{f})$.

transmissivities $T_{i}$, with $1 \leq i \leq 5$. To confirm our hypothesis that parameters are better determined for large velocities (section 6.2), we investigate here two generic cases, which provide two extreme cases of possible crossborehole flow observations (large flow velocity and zero flow velocity). First, we allow only one flow paths between the boreholes ("Test 5," Figure 9b). In the second case, we consider three independent main flow paths
("Test 6," Figure 9b). The corresponding parameter sets are $\left(\log T_{1}, \log T_{2}, \log T_{3}, \log T_{4}, \log T_{5}\right)=(-6,-6,-3$, $-6,-6)$ ("Test 5") and $\left(\log T_{1}, \log T_{2}, \log T_{3}, \log T_{4}\right.$, $\left.\log T_{5}\right)=(-3,-6,-3,-6,-3)$ ("Test 6"). For these examples, the borehole radius is fixed at $0.1 \mathrm{~m}$ and the pumping rate is fixed at $Q=1 \cdot 10^{-2} \mathrm{~m}^{3} / \mathrm{s}$. As before, the transmissivity of the horizontal fractures is set at $T_{B}=5 \cdot 10^{-4} \mathrm{~m}^{2} / \mathrm{s}$.
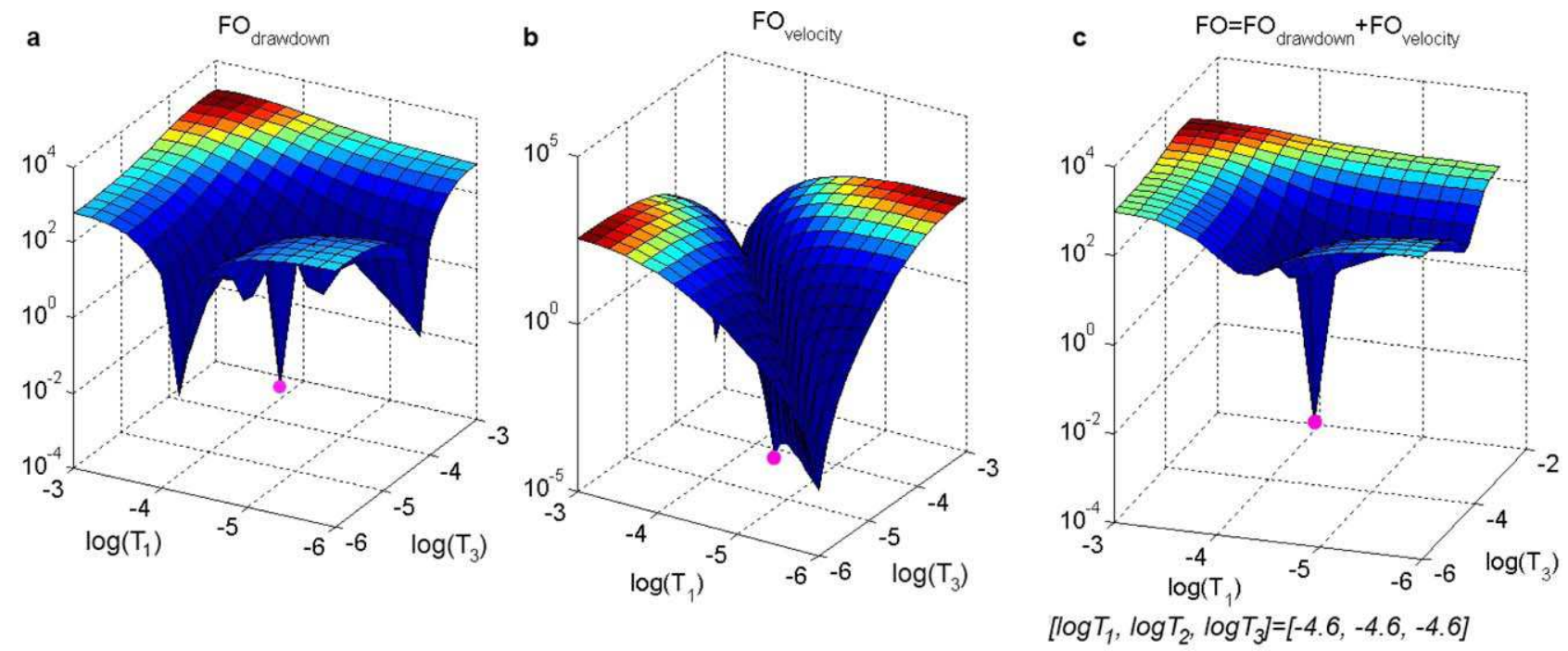

Figure 8. The objective function for the simple synthetic case $\left(\log T_{1}, \log T_{2}, \log T_{3}\right)=$ $(-4.6,-4.6,-4.6)$ (Figure 2) for the (a) drawdown, (b) velocity, and (c) the sum of both with respect to the $\log$-transformed upper and lower connection transmissivities $\left(\log T_{1}\right.$ and $\left.\log T_{3}\right)$. 


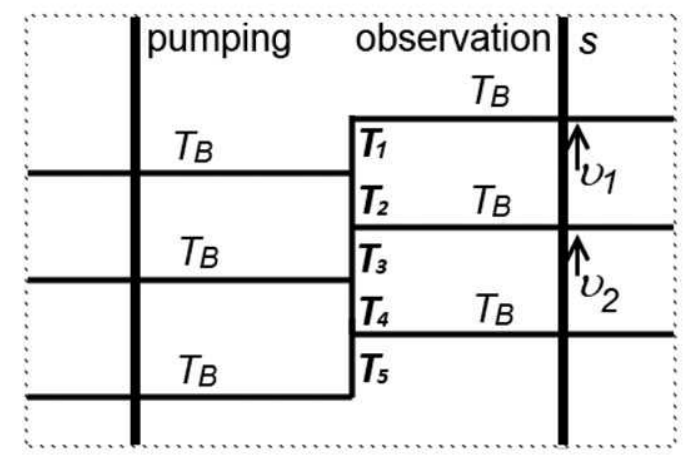

A
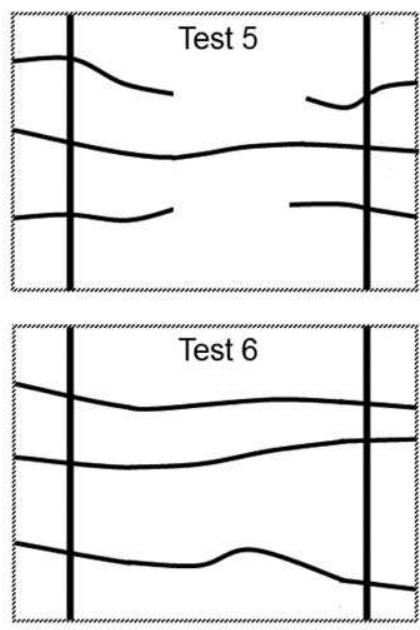

B

Figure 9. Sketch of the synthetic flow model (a) Cross sections of the synthetic flow model. The model comprises six horizontal fractures of transmissivity $T_{B}$ and five vertical fractures of $T_{1}, T_{2}, T_{3}, T_{4}$, and $T_{5}$ transmissivity. (b) Configurations of synthetic tests: "Test 5 " $\left(\log T_{1}, \log T_{2}, \log T_{3}, \log T_{4}, \log T_{5}\right)=$ $(-6,-6,-3,-6,-6)$ and "Test 6 " $\left(\log T_{1}, \log T_{2}, \log T_{3}, \log T_{4}, \log T_{5}\right)=(-3,-6,-3,-6,-3)$.

[40] To probe the interest of performing a tomography experiment, we inverse the "pumping-observation" points for each cross-borehole pumping test. Moreover, we also perform a joint inversion of both "pumping-observation" pumping tests. Observations for the two pumping tests consist of four vertical velocities and two drawdowns in the observation borehole. A set of 50 random starting points is generated for each configuration to search for the minimum in the basins of attraction of the starting points. The minimum of the objective function provides the "true" parame- ter set for all tested cases, including "Test 5" and "Test 6" for each pair of pumping and observation boreholes and for both jointly inverted. Hence, this validates our methodology, and shows that the information content of the data is sufficient to drive the inverse algorithm to the solution. Below we perform the uncertainty analysis for both cases. 6.3.2.1. Complex Synthetic Case, "Test 5"

[41] The distribution of hydraulic heads in the fracture network for "Test 5" is shown in Figure 10a. In this case only one of the fracture connections is significantly

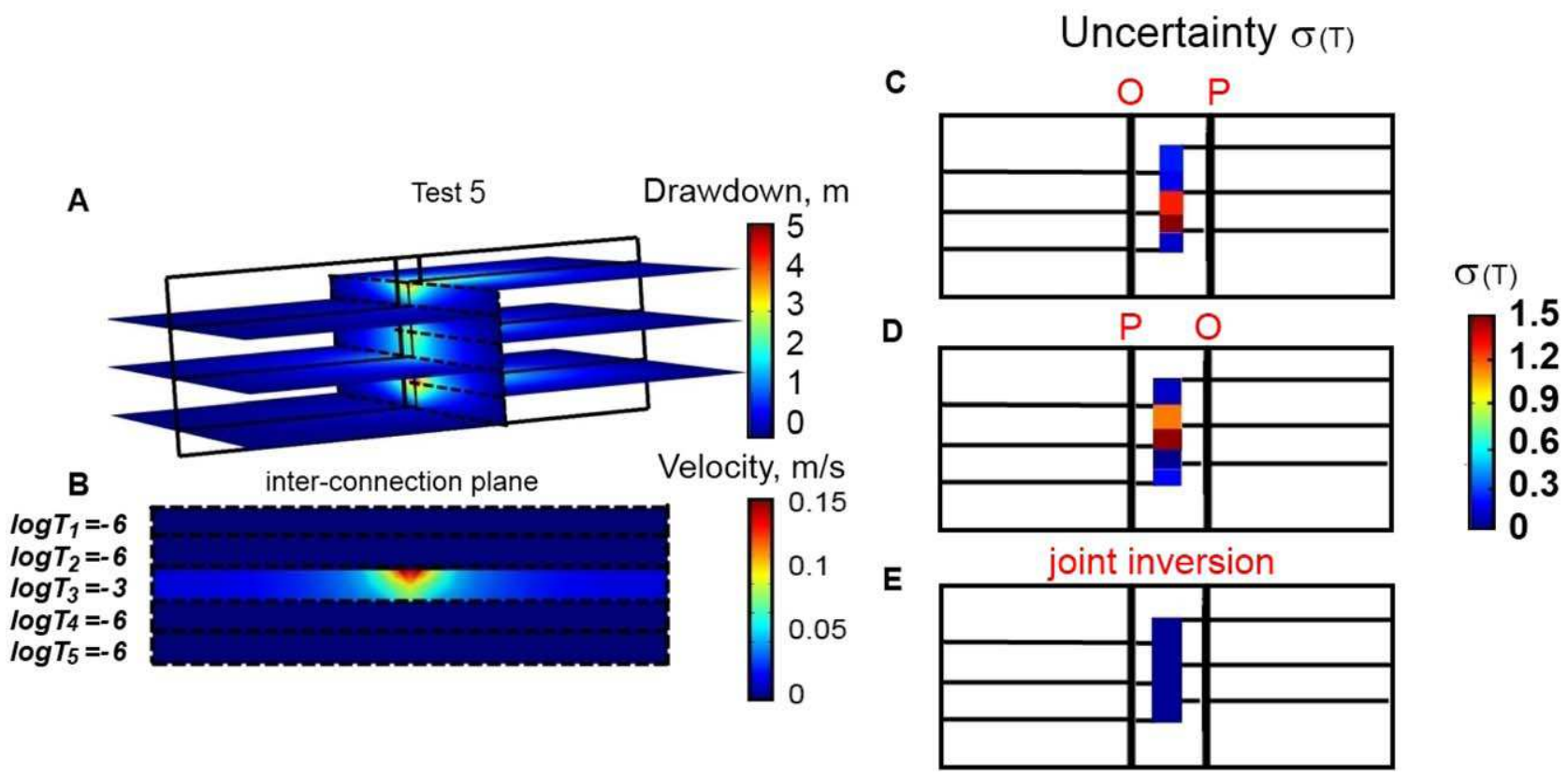

Figure 10. Modeling results for "Test 5." (a) Drawdown distribution for "Test 5" during pumping from the right borehole. (b) Fracture velocities in the interconnection plane. The uncertainty about the estimated log-transformed $T_{1}, T_{2}, T_{3}, T_{4}$, and $T_{5}$ transmissivities (c) during pumping from the right borehole, (d) during pumping from the left borehole, and (e) joint inversion of both pumping tests. 
permeable (Figure 10b). During pumping in one of the wells, the hydraulic head changes in the central connected fracture zone, cause large borehole flow velocities in both borehole sections below and above this flow path in the observation well. The uncertainties in parameter estimation for "Test 5" are shown in Figure 10. Figures 10c and 10d present results of the inversion for each pumping test. High uncertainty about the $T_{3}$ and $T_{4}$ parameters are obtained when pumping from the "right" borehole, as well as about the $T_{2}$ and $T_{3}$ parameters when pumping from the "left" borehole. This is explained by the fact that different combinations of fracture connection transmissivities can provide similar velocities and drawdowns. For instance, during pumping from the "right" borehole, the set of parameters $(-6,-6,-6,-3,-6)$ gives the same observation values as a "true" parameter set $(-6,-6,-3,-6,-6)$. Similarly during pumping from the "left" borehole the set of parameters $(-6,-6,-3,-6,-6)$ and $(-6,-3,-6,-6,-6)$ give identical observation values (Figure 9a). However, joint inversion of the two pumping tests (Figure 10c) leads to a much lower uncertainty. This demonstrates the interest of performing tomography experiments.

\subsubsection{Complex Synthetic Case, "Test 6"}

[42] The distribution of heads and flow velocities for the second synthetic case is shown in Figures 11a and 11b. For "Test 6" the drawdown is large and the velocity is close to zero. As for synthetic case 4 (Figure 2b), zero velocity is caused by equal transmissivity flow paths. The sensitivity analysis performed for synthetic case 4 suggests that estimation of interconnection transmissivities in this case is uncertain. Figures $11 \mathrm{c}-11 \mathrm{e}$ show the uncertainties in parameter estimation for "Test 6." As expected, the inversion results show that small observed velocities do not provide a strong constraint for the interconnection fracture transmissivities $\left(T_{2}\right.$ and $\left.T_{4}\right)$. In this case, the joint inversion of both pairs of pumping and observation wells, do not improve the estimations significantly, and estimated transmissivities of interconnection fracture vary over more than one order of magnitude. Nevertheless, despite this high uncertainty, the inverse model algorithm does converge to the "true" parameter values for the global optimum.

\section{Conclusions}

[43] The flow tomography approach is proposed to characterize the connectivity and transmissivity of preferential permeable flow paths in fractured aquifers. We explore the potential of this approach for simplified synthetic fracture network models and quantify the sensitivity of drawdown and borehole flow velocities to the transmissivity of the connecting flow paths. Flow tomography is expected to be most effective if cross-borehole pumping induces large changes in vertical borehole velocities. The uncertainty of the transmissivity estimates increases for small borehole flow velocities. The uncertainty about the transmissivity of fractures that connect the main flow path but not the boreholes is generally higher.

[44] An inverse model approach is developed to estimate log-transformed transmissivity values of hydraulically active fractures between the pumping and observation wells by inverting cross-borehole flow and water level data. While the misfit functions for drawdown data alone are characterized by multiple minima, the global objective function for drawdown and flow velocity is convex and
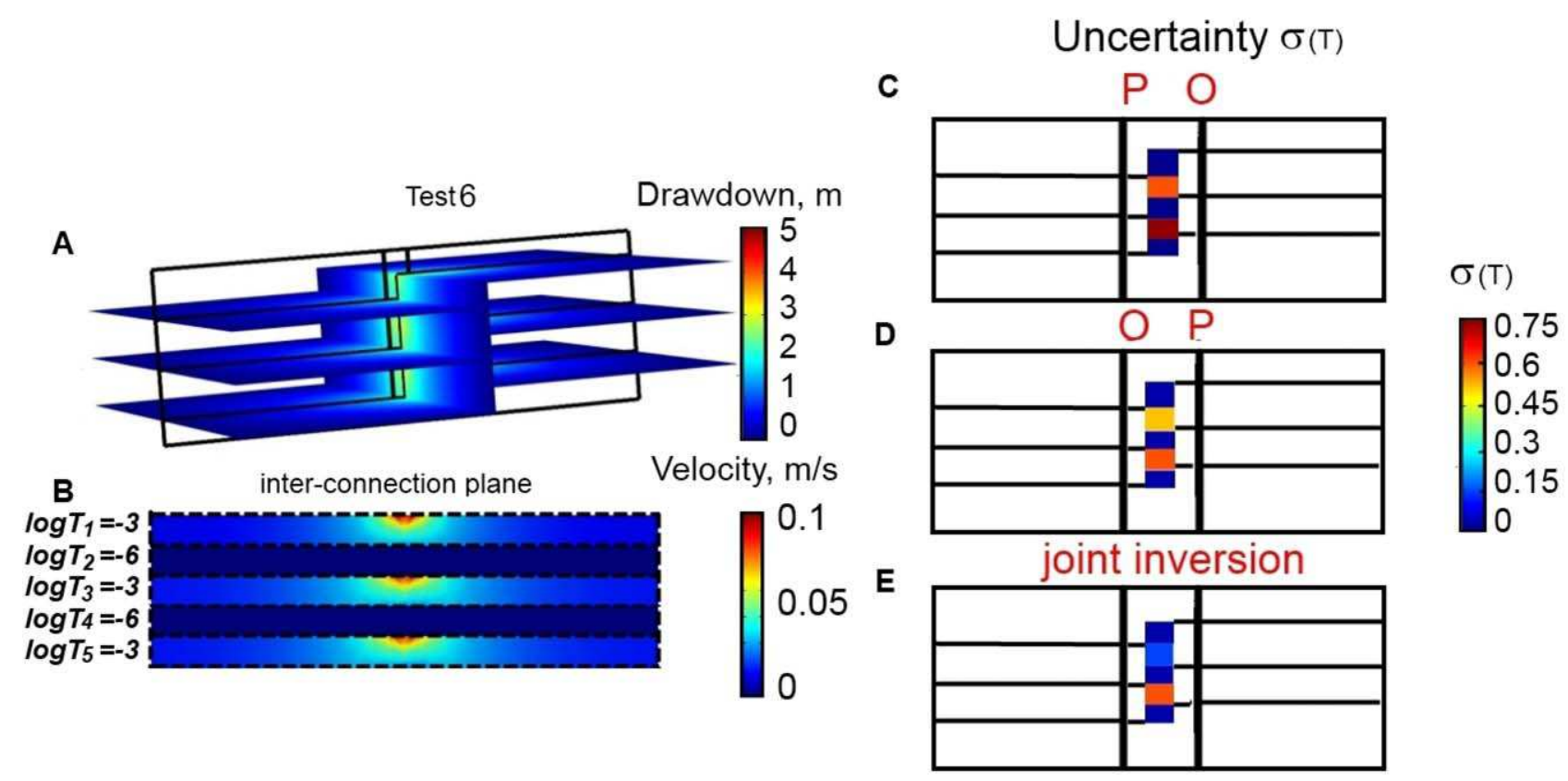

Figure 11. Modeling results for "Test 6." (a) Drawdown distribution for "Test 6" during pumping from the right borehole. (b) Fracture velocities in the interconnection plane. Uncertainty about the estimated log-transformed $T_{1}, T_{2}, T_{3}, T_{4}$, and $T_{5}$ transmissivities (c) during pumping from the left borehole, (d) during pumping from the right borehole, and (e) joint inversion of both pumping tests. 
possesses a global minimum. Thus, the inverse model is shown to converge to the "true" parameter values in all tested cases. Our analysis also demonstrates that the inversion of pumping and observation points significantly reduces the uncertainty about parameter estimation. These conclusions are confirmed for more complex connectivity patterns.

[45] Flow tomography appears to be a promising approach for identification of general connectivity patterns and transmissivities of the main flow paths. Even though the chosen fracture network geometry has been simplified here, the general methodology may be applied to other fracture network geometries. Hence, the results of this investigation encourage the application of flow tomography to natural fractured aquifers. Furthermore, a possible extension of this inverse approach consists of using transient flow data to estimate both transmissivities and specific storage of the main flowing fractures.

[46] Acknowledgments. This work was supported by the European Marie Curie network IMVUL (grant agreement 212298), by the National Research Observatory $\mathrm{H}+$ and by the European Interreg IV project CLIMAWAT.

\section{References}

Bonnet, E., O. Bour, N. E. Odling, P. Davy, I. Main, P. Cowie, and B. Berkowitz (2001), Scaling of fracture systems in geological media, Rev. Geophys., 39(3), 347-383.

Brauchler, R., R. Hu, P. Dietrich, and M. Sauter (2011), A field assessment of high-resolution aquifer characterization based on hydraulic travel time and hydraulic attenuation tomography, Water Resour. Res., 47, W03503, doi:10.1029/2010WR009635.

Butler, J. J., McElwee, C. D., and Bohling, G. C. (1999), Pumping tests in networks of multilevel sampling wells: Motivation and methodology, Water Resour. Res., 35(11), 3553-3560.

Castagna, M., M. W. Becker, and A. Bellin (2011), Joint estimation of transmissivity and storativity in a bedrock fracture, Water Resour. Res., 47, W09504, doi:10.1029/2010WR009262.

Cheng, J.-M., and W.W.-G. Yeh (1992), A proposed quasi-Newton method for parameter identification in a flow and transport system, Adv. Water Resour., 15(4), 239-249.

Day-Lewis, F. D., P. A. Hsieh, and S. M. Gorelick (2000), Identifying fracture-zone geometry using simulated annealing and hydraulicconnection data, Water Resour. Res., 36(7), 1707-1721, doi:10.1029/ 2000WR900073.

Day-Lewis, F. D., J. W. Lane Jr., J. M. Harris, and S. M. Gorelick (2003), Time-lapse imaging of saline-tracer transport in fractured rock using difference-attenuation radar tomography, Water Resour. Res., 39(10), 1290, doi:10.1029/2002WR001722.

Day-Lewis, F. D., K. Singha, and A. M. Binley (2005), Applying petrophysical models to radar travel time and electrical resistivity tomograms: Resolution-dependent limitations, J. Geophys. Res., 110, B08206, doi: 10.1029/2004JB003569.

Dorn, C., N. Linde, T. Le Borgne, O. Bour, and L. Baron (2011), Singlehole GPR reflection imaging of solute transport in a granitic aquifer, Geophys. Res. Lett., 38, L08401, doi:10.1029/2011GL047152.

Dorn, C., N. Linde, T. Le Borgne, O. Bour, and M. Klepikova (2012), Inferring transport characteristics in a fractured rock aquifer by combining single-hole GPR reflection monitoring and tracer test data, Water Resour. Res., 48, W11521, doi:10.1029/2011WR011739.

Hao, Y., T. C. Yeh, J. Xiang, W. A. Illman, K. Ando, K. C. Hsu, and C. H. Lee (2008), Hydraulic tomography for detecting fracture zone connectivity, Ground Water, 46(2), 183-192, doi:10.1111/j.1745-6584.2007.00388.x.

Hess, A. E. (1986), Identifying hydraulically conductive fractures with a slow velocity borehole flowmeter, Can. Geotechnol., 23, 69-78.
Hill, M. C., and C. R. Tiedeman (2007), Effective Groundwater Model Calibration: With Analysis of Data, Sensitivities, Predictions, and Uncertainty, pp. 383-390, John Wiley, New York.

Illman, W. A., A. J. Craig, and X. Liu (2008), Practical issues in imaging hydraulic conductivity through hydraulic tomography, Ground Water, 46(1), 120-132.

Illman, W. A., X. Liu, S. Takeuchi, T.-C. J. Yeh, K. Ando, and H. Saegusa (2009), Hydraulic tomography in fractured granite: Mizunami Underground Research site, Japan, Water Resour. Res., 45, W01406, doi:10.1029/2007WR006715.

Klepikova, M., T. Le Borgne, O. Bour, and P. Davy (2011), A methodology for using temperature-depth profiles under ambient, single and crossborehole pumping conditions to estimate fracture hydraulic properties, $J$. Hydrol., 407(1-4), 145-152, doi:10.1016/j.jhydrol.2011.07.018.

Le Borgne, T., F. L. Paillet, O. Bour, and J.-P. Caudal (2006a), Cross-borehole flowmeter tests for transient heads in heterogeneous aquifers, Ground Water, 44, 444-452, doi:10.1111/j.1745-6584.2005.00150.x.

Le Borgne, T., O. Bour, F. L. Paillet, and J.-P. Caudal (2006b), Assessment of preferential flow path connectivity and hydraulic properties at singleborehole and cross-borehole scales in a fractured aquifer, J. Hydrol., 328, 347-359, doi:10.1016/j.jhydrol.2005.12.029.

Le Borgne, T., et al. (2007), Comparison of alternative methodologies for identifying and characterizing preferential flow paths in heterogeneous aquifers, J. Hydrol., 345(3-4), 134-148, doi:10.1016/j.jhydro1.2007.07.007.

Le Goc, R., J.-R. de Dreuzy, and P. Davy (2010), An inverse problem methodology to identify flow channels in fractured media using synthetic steady-state head and geometrical data, Adv. Water Resour., 33(3-4), 782-800, doi:10.1016/j.advwatres.2010.04.011.

Mayer, A. S., and C. Huang (1999), Development and application of a coupled-process parameter inversion model based on the maximum likelihood estimation method, Adv. Water Resour., 22(8), 841-853.

Molz, F. J., R. H. Morin, A. E. Hess, J. G. Melville, and O. Guven (1989), The impeller meter for measuring aquifer permeability variations: Evaluation and comparison with other tests, Water Resour. Res., 25(7), 1677-1683.

Olsson, O. (1992), Site characterization and validation, Final Rep., Stripa Project 92-22, Conterra AB, Uppsala, Sweden.

Paillet, F. L. (1998), Flow modeling and permeability estimation using borehole flow logs in heterogeneous fractured formations, Water Resour. Res., 34(5), 997-1010.

Paillet, F. L. (2000), A field technique for estimating aquifer parameters using flow log data, Ground Water, 38(4), 510-521.

Paillet, F. L., J. H. Williams, J. Urik, J. Lukes, M. Kobr, and S. Mares (2012), Cross-borehole flow analysis to characterize fracture connections in the Melechov Granite, Bohemian-Moravian Highland, Czech Republic, Hydrogeol. J., 20(1), 143-154, doi:10.1007/s10040-011-0787-1.

Sawdey, J. R., and A. S. Reeve (2012), Automated inverse computer modeling of borehole flow data in heterogeneous aquifers, Comput. Geosci., 46, 219-228.

Sharmeen, R., W. A. Illman, S. J. Berg, T.-C. J. Yeh, Y.-J. Park, E. A. Sudicky, and K. Ando (2012), Transient hydraulic tomography in a fractured dolostone: Laboratory rock block experiments, Water Resour. Res., 48 , W10532, doi:10.1029/2012WR012216.

Tarantola, A. (2004), Inverse Problem Theory and Methods for Model Parameter Estimation, Soc. for Ind. and Appl. Math., Philadelphia, Pa.

Tsang, C. F., and I. Neretnieks (1998), Flow channelling in heterogeneous fractured rocks, Rev. Geophys., 36(2), 275-298.

Wellman, T. P., and E. P. Poeter (2005), Estimating spatially variable representative elementary scales in fractured architecture using hydraulic head observations, Water Resour. Res., 41, W03001, doi:10.1029/2004WR003287.

Williams, J. H., and F. L. Paillet (2002), Using flowmeter pulse tests to define hydraulic connections in the subsurface: A fractured shale example, J. Hydrol., 265(1-4), 100-117.

Yeh, T.-C. J., and S. Liu (2000), Hydraulic tomography: Development of a new aquifer test method, Water Resour. Res., 36(8), 2095-2105, doi:10.1029/2000WR900114.

Yeh, W. W.-G. (1986), Review of parameter identification procedures in groundwater hydrology: The inverse problem, Water Resour. Res., 22(2), 95-108.

Ugray, Z., L. Lasdon, J. C. Plummer, F. Glover, J. Kelly, and R. Marti (2007), Scatter search and local NLP solvers: A multistart framework for global optimization, INFORMS J. Comput., 19(3), 328-340. 